\title{
The Fundamental Role of In Situ Electron Microscopy in Catalytic Science
}

\author{
P.A. Crozier* \\ *Center for Solid State Science, Arizona State University, Tempe, AZ 85287-1704
}

It is widely recognized that alternative sources of environmentally clean energy must be developed in order to avert the possible negative consequences associated with global climate change and decreasing oil production. The demand for efficient, clean energy will require a dramatic improvement in our ability to synthesis new fuels, maximize fuel utilization efficiency and minimize detrimental effects on the environment. The synthesis of new heterogeneous catalysts will be critical for all stages of the development of a new energy infrastructure. This will require significant improvements in our understanding of the catalyst structure-activity relationship in order to design and control the synthesis of suitable nanostructures with the desired catalytic activity.

In situ environmental transmission electron microscopy (ETEM) is a powerful tool for exploring gas-solid reactions at the atomic level. The extensive pioneering work of Baker [1] and Gai [2] showed that ETEM is ideal for visualizing nanoscale features under near-reactor conditions on the high surface area supports employed in heterogeneous catalysts. We have successfully used ETEM to explore many aspects of catalytic function including synthesis of supported metal particles [3], polymerization [4], redox processes [5,6], de-activation and regeneration processes [7]. In the modern ETEM, the combination of atomic resolution imaging and nanospectroscopy provides dynamic information on both the structural and compositional transformations that take place under reactive gas conditions. This allows us to address some fundamental questions concerning the response of the catalyst to reactive gases and the relationship between surface structures and catalytic activity. Combining this information with data from other techniques including density functional theory allows us to develop a complete description of the reaction steps taking place on the surface of the catalyst. Consequently we are able to identify the most active surface sites thus providing parameters for the design and synthesis of catalysts for particular applications.

To control the active sites on the surface of the catalyst it is necessary to have a very high level of control over the catalyst synthesis. Many techniques for making supported metal catalysts, including the frequently employed impregnation approaches, involve self-assembly steps. During impregnation synthesis, metal containing salts dry on the high surface area support, get thermally decomposed and metal species diffuse over the support and eventually assemble into metal nanoparticles. There is wide recognition of the importance of understanding and controlling this process to improve the performance of the final catalysts. However, little is known about the detailed role of the nanoscale decomposition, diffusion, nucleation and growth processes taking place during precursor reduction. We are employing ETEM to study all steps in this complex and interconnected process so that we can improve our control of the catalyst synthesis process [3]. Results from bimetallic synthesis are presented in these proceedings [8].

Identifying the phase with the highest catalytic activity is an important goal for ETEM. We are currently using ETEM to develop an understanding of the relationship between catalytic activity and structure for ceria zirconia based oxides [9]. In these systems, the catalytic activity for gas oxidation and reduction is directly related to the ability of ceria to donate or remove oxygen from the crystal surface during a gas-solid reaction. In this case, when oxygen is removed from the crystal it causes the Ce cation to undergo a transition from the $\mathrm{Ce}^{+4}$ to $\mathrm{Ce}^{+3}$ oxidation state. The ease with which this transition takes place is dependent on the details of the local atomic environment around 
the $\mathrm{Ce}$ in ways that are not yet fully understood. For example, material showing nominally similar structure may become much more active after exposure to a severe reduction and re-oxidation cycle. The structural change that brings about enhanced activity is subtle and is not presently understood. In situ electron microscopy can play an important role in solving this problem because it permits the redox activity of individual nanocrystals to be determined allowing more active phases to be identified. This approach is illustrated in the example below. A $\mathrm{Ce}_{0.5} \mathrm{Zr}_{0.5} \mathrm{O}_{2}$ catalyst that shows desirable low-temperature redox behavior was loaded into the ETEM and heated in 1 Torr of $\mathrm{H}_{2}$ to about $575^{\circ} \mathrm{C}$. In situ electron energy-loss spectroscopy was used to determine the oxidation state of the $\mathrm{Ce}$ and determine when reduction occurred (by observing changes in the relative intensity of the white lines at the beginning of the $\mathrm{Ce}_{45}$ edge). The EEL spectra in Figure 1 show that these two nominally identical particles from the same batch of catalyst have very different redox properties; one nanocrystal is reduced and the other remains fully oxidized. In this case, the reduced crystal would certainly be the more active component of the catalyst at low temperatures. This variation in behavior provides us with an opportunity to determine the critical structural features that give rise to the difference in catalytic activity. At present we are conducting a detailed study of this phenomenon in order to understand the critical structural changes that lead to enhanced low temperature activity [9].

\section{References}

[1] Baker, R.T.K., and Chludzinski, J.J., J.Phys. Chem., 90, (1986), 4734.

[2] Gai, P.L., Topics in Catalysis, 8 (1999) 97.

[3] Li, P. et al, J.Chem.Phys. B, 109, (2005) 13883.

[4] Oleshko, V.P. et al, Journal of Electron Microscopy, 51 (2002), S27.

[5] Crozier, P.A. and A.K. Datye, Studies in Surface Science and Catalysis, 130, (2000), 3119.

[6] Sharma, R. et al, Phil. Mag. 84, (2004), 2731.

71. Liu, R.-J. et al, Applied Catalysis A-General, 282, (2005), 111.

8. Li, P. et al, (these proceedings).

9] Wang, R. et al, (these proceedings).

[10] The support from the National Science Foundation (NSF-CTS-0306688) and the use of TEM at the John M.Cowley Center for High Resolution Microscopy at Arizona State University are gratefully acknowledged.

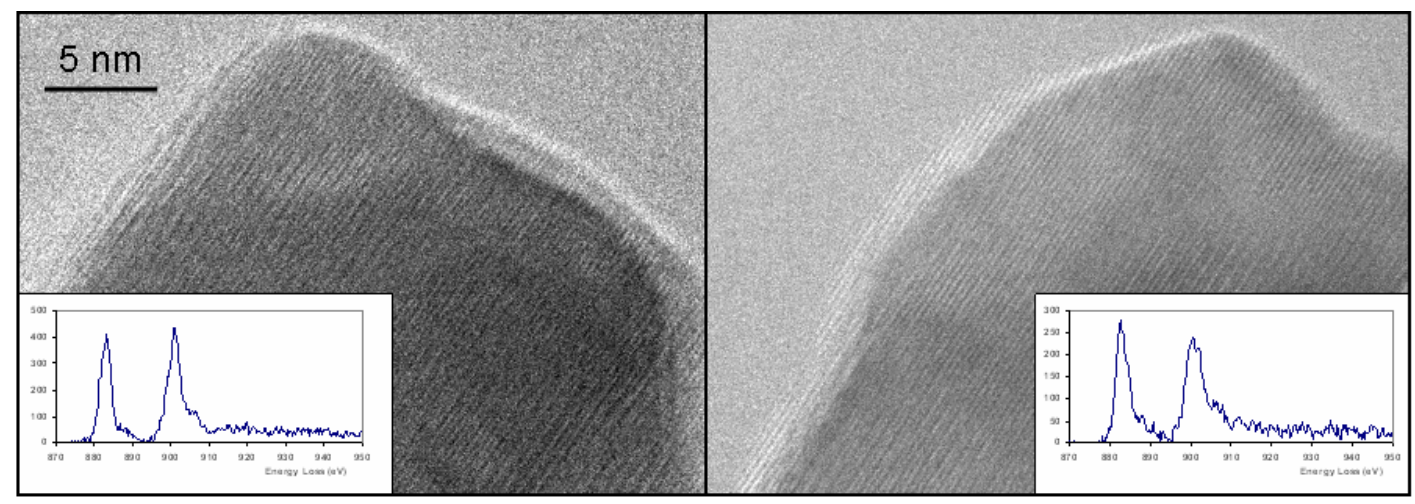

Figure 1: In situ HREM images and energy loss spectra from nominally identical nanoparticles of $\mathrm{Ce}_{0.5} \mathrm{Zr}_{0.5} \mathrm{O}_{2}$ recorded at $575^{\circ} \mathrm{C}$ in 1 Torr of $\mathrm{H}_{2}$. The in situ EELS (inserts) show that the particle on the left is unreduced while the particle on the right is reduced. 